\title{
Restoration of hepatic glycogen deposition reduces hyperglycaemia, hyperphagia and gluconeogenic enzymes in a streptozotocin-induced model of diabetes in rats
}

\author{
S. Ros • M. García-Rocha • J. Calbó • J. J. Guinovart
}

Received: 14 March 2011 /Accepted: 10 June 2011 /Published online: 3 August 2011

(C) Springer-Verlag 2011

\begin{abstract}
Aims/hypothesis Glycogen deposition is impaired in diabetes, thus contributing to the development of hyperglycaemia. Several glucose-lowering strategies have attempted to increase liver glycogen deposition by modulating targets, which eventually trigger the activation of liver glycogen synthase (LGS). However, these targets also alter several other biological processes, and therefore their therapeutic use may be limited. Here we tested the approach of directly activating LGS and evaluated the potential of this strategy as a possible treatment for diabetes.

Methods In this study, we examined the efficacy of directly overproducing a constitutively active form of LGS in the liver to ameliorate streptozotocin-induced diabetes in rats. Results Activated mutant LGS overproduction in the liver of streptozotocin-induced diabetic rats normalised liver glycogen content, despite low levels of glucokinase and circulating insulin. Moreover, this overproduction led to a decrease in food intake and in the production of the main gluconeogenic
\end{abstract}

S. Ros · M. García-Rocha · J. Calbó · J. J. Guinovart $(\bowtie)$

Institute for Research in Biomedicine (IRB Barcelona),

Baldiri Reixac 10,

08028 Barcelona, Spain

e-mail: guinovart@irbbarcelona.org

S. Ros · M. García-Rocha · J. Calbó · J. J. Guinovart Centro de Investigación Biomédica en Red de Diabetes y

Enfermedades Metabólicas Asociadas (CIBERDEM),

Barcelona, Spain

S. Ros $\cdot$ J. J. Guinovart

Department of Biochemistry and Molecular Biology,

University of Barcelona,

Barcelona, Spain

Present Address:

S. Ros

London Research Institute-Cancer Research UK,

London, UK enzymes, glucose-6-phosphatase, fructose-1,6-bisphosphatase and phosphoenolpyruvate carboxykinase. The resulting combined effect was a reduction in hyperglycaemia.

Conclusions/interpretation The restoration of liver glycogen ameliorated diabetes and therefore is considered a potential strategy for the treatment of this disease.

Keywords Glycogen · Diabetes · Food intake ·

Gluconeogenesis · Glucose homoeostasis .

Hyperglycaemia - Liver Liver glycogen synthase .

Metabolism

\begin{tabular}{|c|c|}
\hline \multicolumn{2}{|c|}{ Abbreviations } \\
\hline FBPase & Fructose-1,6-bisphosphatase \\
\hline Bgal & $\beta$-Galactosidase \\
\hline GK & Glucokinase \\
\hline GP & Glycogen phosphorylase \\
\hline G6P & Glucose 6-phosphate \\
\hline G6Pase & Glucose-6-phosphatase \\
\hline GS & Glycogen synthase \\
\hline GSK-3 & Glycogen synthase kinase 3 \\
\hline LGS & Liver glycogen synthase \\
\hline $\mathrm{PC}$ & Pyruvate carboxylase \\
\hline PEPCK & Phosphoenolpyruvate carboxykinase \\
\hline PP1 & Protein phosphatase 1 \\
\hline PTG & Protein targeting to glycogen \\
\hline Q-PCR & Quantitative PCR \\
\hline STZ & Streptozotocin \\
\hline wt & Wild-type \\
\hline
\end{tabular}

\section{Introduction}

Postprandial glucose is cleared from blood mainly by conversion into glycogen in liver and skeletal muscle. In mammals there are two glycogen synthase (GS) isoforms, 
namely liver GS (LGS, coded by the Gys2 gene), the production of which is tissue-specific, and muscle GS (coded by the Gys 1 gene), which is produced in other tissues [1]. Muscle glycogen provides a local source of energy, while liver glycogen is converted into glucose and released into the bloodstream to prevent hypoglycaemia during the early stages of fasting. In diabetes, the accumulation of liver glycogen is impaired, thereby contributing to the development of hyperglycaemia [2-4]. Recently, several proteins involved in the control of hepatic glycogen metabolism, including glucokinase (GK), glycogen phosphorylase (GP) and glycogentargeting proteins, have been studied as potential targets for anti-hyperglycaemic therapy, although there are several concerns associated with their modulation. GK overproduction increases flux through glycolysis and, in some circumstances, this can lead to an increase in plasma triacylglycerols [5]. GP inhibition may have potentially negative effects on skeletal muscle function during exercise [6]. Also, studies using glycogen-targeting proteins have shown that sustained efficacy in improving glucose tolerance can be accomplished only by enhancing glycogen synthesis in the postprandial state without compromising glycogenolysis in the postabsorptive state [7, 8]. Moreover, there has been recent interest in selective GS kinase 3 (GSK-3) inhibitors as hypoglycaemic agents. A common feature of all the above-mentioned strategies is that they lead to secondary activation of LGS. We have previously reported that glycogen synthesis in primary cultured hepatocytes is enhanced by the production of constitutively active forms of LGS [9], in which the inactivating phosphorylation of seven serine residues is prevented by mutation into alanine residues either singly or in pairs. Moreover, we have recently shown that the overproduction of one of these constitutively active LGS mutant forms in the liver of healthy animals is an effective method for improving glucose tolerance in the postprandial state, as a result of its capacity to enhance glucose storage without affecting other metabolic pathways [10]. In the light of these encouraging findings, the current study addressed the overproduction of this constitutively active LGS mutant form in liver of rats with streptozotocin (STZ)-induced diabetes. Thus, here we further studied the effects of this mutant in liver glycogen metabolism in a diabetic animal model, and also whether it affects other metabolic pathways that are altered in the diabetic state. We found that restoration of hepatic glycogen was associated with reductions in hyperphagia, the production of the main gluconeogenic enzymes, and hyperglycaemia.

\section{Methods}

Preparation of recombinant adenoviruses Recombinant purified adenoviruses encoding the bacterial enzyme $\beta$ - galactosidase ( $\beta \mathrm{gal})$, Rattus norvegicus wild-type LGS (wt-LGS) [11] or a constitutively active Rattus norvegicus LGS variant mutated at two phosphorylation sites, sites 2 and $3 b$ (activated mutant-LGS) [9], were amplified and purified for injection into animals using previously described procedures [12]. The above described adenoviruses and recombinant purified adenoviruses encoding the catalytically inactive LGS mutant at Glu509Ala (E509A-LGS) [13] and this catalytically inactive variant additionally mutated at the 2 and $3 \mathrm{~b}$ phosphorylation sites (E509Amutant-LGS) [9] were amplified and purified for hepatocyte infection using previously described procedures.

Animal studies All procedures were approved by Barcelona Science Park's Animal Experimentation Committee and were carried out in accordance with the European Community Council Directive and the National Institute of Health guidelines for the care and use of laboratory animals. Male Wistar rats (Charles River Laboratories, L'Arbresle, France) weighing 200-250 g were housed 1 week before any procedure, and were allowed free access to water and standard laboratory chow (Harlan Teklad Laboratory diet 7001; Harlan Teklad, Gannat, France). After procedures, rats were caged individually under a standard $12 \mathrm{~h} \mathrm{light} / 12 \mathrm{~h}$ dark cycle to allow the monitoring of food intake and water. Rats were injected intraperitoneally with a single moderate dose of STZ (2deoxy-2-(3-methyl-3-nitrosourea)-1-D-glucopyranose; $50 \mathrm{mg} / \mathrm{kg}$; Sigma-Aldrich Quimica SA, Madrid, Spain). Only those animals in which blood glucose rose to concentrations greater than $22 \mathrm{mmol} / \mathrm{l}$ within 6 days after STZ injection were used. Rats were anaesthetised with isofluorane $2 \%$ (Isoba vet; Schering Plough-Merck \& Co, Summit, NJ, USA) and infused with $1 \times 10^{12}$ particles of activated mutant-LGS, wt-LGS or $\beta$ gal purified adenoviruses via tail vein injection. One group of healthy animals was also infused with the same amount of $\beta$ gal adenovirus. Two protocols were performed. In the first, animals were allowed to feed ad libitum until $144 \mathrm{~h}$ after viral administration. Food intake was measured during three successive $24 \mathrm{~h}$ periods, beginning 3 days after viral treatment. In the second protocol, animals were starved for the last $18 \mathrm{~h}$. In all cases, after $144 \mathrm{~h}$, rats were anaesthetised with sodium thiopental (Tiobarbital; $0.1 \mathrm{~g} / \mathrm{kg}$ body weight intraperitoneally; B. Braun Medical, Sheffield, UK) and killed for the collection of blood and tissue samples. The samples collected were excised, rapidly snap-frozen in liquid nitrogen, and stored at $-80^{\circ} \mathrm{C}$ for further analysis.

Hepatocyte isolation, culture and treatment with recombinant adenoviruses Collagenase perfusion was used to isolate hepatocytes from male Wistar rats (180-225 g) starved for $24 \mathrm{~h}$, as described previously [14]. Cells were seeded at a final density of $8 \times 10^{4}$ cells $/ \mathrm{cm}^{2}$ onto plastic plates of 
$60 \mathrm{~mm}$ diameter treated with $0.001 \%$ (wt/vol.) collagen solution (Sigma). Media were replaced with fresh DMEM containing $25 \mathrm{mmol} / \mathrm{l}$ glucose and 10\% (vol./vol.) FBS for $12 \mathrm{~h}$. Cells were treated for $3 \mathrm{~h}$ with adenoviruses, at a similar multiplicity of infection, in FBS-free DMEM containing $25 \mathrm{mmol} / 1$ glucose. Media were then replaced with FBS-free DMEM containing $25 \mathrm{mmol} / 1$ glucose, in the absence or presence of $100 \mathrm{nmol} / 1$ dexamethasone and $0.3 \mathrm{mmol} / 1 \mathrm{dibu}-$ tyryl cAMP, and another incubation of $24 \mathrm{~h}$ was performed. At the end of each manipulation, cell monolayers were flashfrozen in liquid $\mathrm{N}_{2}$ and stored at $-80^{\circ} \mathrm{C}$ until analysis.

Metabolite determinations and enzyme activity assays To measure GS activity in tissue, $1 \mathrm{ml}$ ice-cold homogenisation buffer was added for every $100 \mathrm{mg}$ tissue. Buffer consisted of $10 \mathrm{mmol} / \mathrm{l}$ TRIS/ $\mathrm{HCl}(\mathrm{pH} \mathrm{7}), 150 \mathrm{mmol} / \mathrm{l}$ $\mathrm{KF}, 15 \mathrm{mmol} / 1 \mathrm{EDTA}, 15 \mathrm{mmol} / \mathrm{l}$ 2-mercaptoethanol, $0.6 \mathrm{~mol} / 1$ sucrose, $1 \mathrm{mmol} / 1$ benzamidine, $1 \mathrm{mmol} / 1$ phenylmethanesulphonyl fluoride, $25 \mathrm{nmol} / 1$ okadaic acid, $10 \mu \mathrm{g} / \mathrm{ml}$ leupeptin, $10 \mu \mathrm{g} / \mathrm{ml}$ aprotinin and $10 \mu \mathrm{g} / \mathrm{ml}$ pepstatin. Homogenisation was performed at $4{ }^{\circ} \mathrm{C}$ with a Polytron homogeniser. GS activity was measured in homogenates in the presence or absence of $6.6 \mathrm{mmol} / 1$ glucose 6-phosphate (G6P) [15]. GS activity in the absence of G6P represents the active form of the enzyme, while in the presence of $6.6 \mathrm{mmol} / \mathrm{l} \mathrm{G6P}$, it is a measure of total activity. Liver glycogen content was determined by an amyloglucosidase-based assay, as described elsewhere [16].

Electrophoresis and immunoblotting Immunoreactivity was determined by resolving homogenates ( $20 \mu \mathrm{g}$ protein) by $10 \%$ SDS-PAGE. The protein was transferred onto a nitrocellulose membrane and probed with the following antibodies: a rabbit antibody against LGS [17], a rabbit antibody against GS phosphorylated on Ser640 (p-Ser640 GS; Cell Signaling Technology, Beverly, MA, USA), a rabbit antibody against GK [18], a sheep antibody against phosphoenolpyruvate carboxykinase (PEPCK) (generously given by D. Granner, Vanderbilt University, Nashville, TN, USA), rabbit antibodies against total liver GP or against GP phosphorylated on Ser14 $[19,20]$, a rabbit antibody against total p44/42 mitogenactivated protein kinase (ERK) (Upstate Biotechnology, Waltham, MA, USA) or a mouse antibody against glyceraldehyde-3-phosphate dehydrogenase (Sigma). Secondary antibodies conjugated to horseradish peroxidase against rabbit (Amersham/GE Healthcare, Piscataway, NJ, USA), mouse (DakoCytomation, Glostrup, Denmark) or sheep (DakoCytomation) immunoglobulins were used. Immunoreactive bands were visualised using an ECLplus kit (GE Healthcare), following the manufacturer's instructions.

Blood variables Blood glucose was measured using a HemoCue Glucose Analyzer (HemoCue, Angelholm,
Sweden). The rest of the variables were measured in plasma. Lactate (HORIBA ABX, Montpellier, France) and triacylglycerol (Sigma) levels were measured spectrophotometrically by standard techniques adapted to a Cobas Mira Analyzer. Rat insulin and leptin levels were measured by immunoassay using EIA kits (Spi Bio, Paris, France), following the manufacturer's instructions.

RNA purification and analysis Total RNAwas isolated from rat liver tissue by homogenising a $100 \mathrm{mg}$ sample in $1 \mathrm{ml}$ TRIzol reagent as described previously [10]. Then $5 \mu \mathrm{g}$ total RNA from each sample was reverse-transcribed for $50 \mathrm{~min}$ at $42^{\circ} \mathrm{C}$ in a $15 \mu \mathrm{l}$ reaction volume using $200 \mathrm{U}$ SuperScript III reverse transcriptase (SuperScript First-strand Synthesis System for RT-Q-PCR; Invitrogen, Barcelona, Spain) in the presence of $50 \mathrm{ng}$ random hexamers. Quantitative real-time PCR (Q-PCR) tests were performed following the standard protocol of the ABI Prism 7900 Detection System together with the appropriate ready-made TaqMan primer/probe sets (Applied Biosystems, Madrid, Spain) at the Genomic Unit of the Core Scientific Services at the University of Barcelona. Each sample was analysed in three replica wells with $30 \mathrm{ng}$ first-strand cDNA in a total reaction volume of $10 \mu \mathrm{l}$. The temperature profile consisted of 40 cycles of $15 \mathrm{~s}$ at $95^{\circ} \mathrm{C}$ and $1 \mathrm{~min}$ at $60^{\circ} \mathrm{C}$. Data were analysed with the $2^{-\Delta \Delta \mathrm{C}_{\mathrm{t}}}$ method using $18 \mathrm{~S}$ rRNA as endogenous control.

Statistical analysis Data are expressed as the mean $\pm \mathrm{SE}$. Statistical significance was determined by unpaired Student's $t$ test using Microsoft Excel (version XP; Microsoft, Redmond, WA, USA). Statistical significance was assumed at $p \leq 0.05$.

\section{Results}

Production of wt-LGS and activated mutant-LGS forms in liver of STZ-treated rats Six days after STZ administration, animals with blood glucose concentrations above $22 \mathrm{mmol} / \mathrm{l}$ were injected with purified adenovirus encoding wt-LGS [11], activated mutant-LGS or $\beta$ gal via the tail vein, which has been shown to produce an hepatic preferential transgene delivery [21, 22]. In addition, a group of healthy rats was injected with adenovirus codifying $\beta$ gal. The efficiency of adenovirus-mediated transduction of LGS in the liver was confirmed by the significant increase in mRNA expression (Fig. 1a), total GS activity (Fig. 1b) and LGS immunoreactivity (Fig. 1d) in liver overproducing wt-LGS or the activated mutantLGS, compared with $\beta$ gal-overproducing rats. However, active GS (measured in the absence of G6P) was only markedly increased in the activated mutant-LGS group 

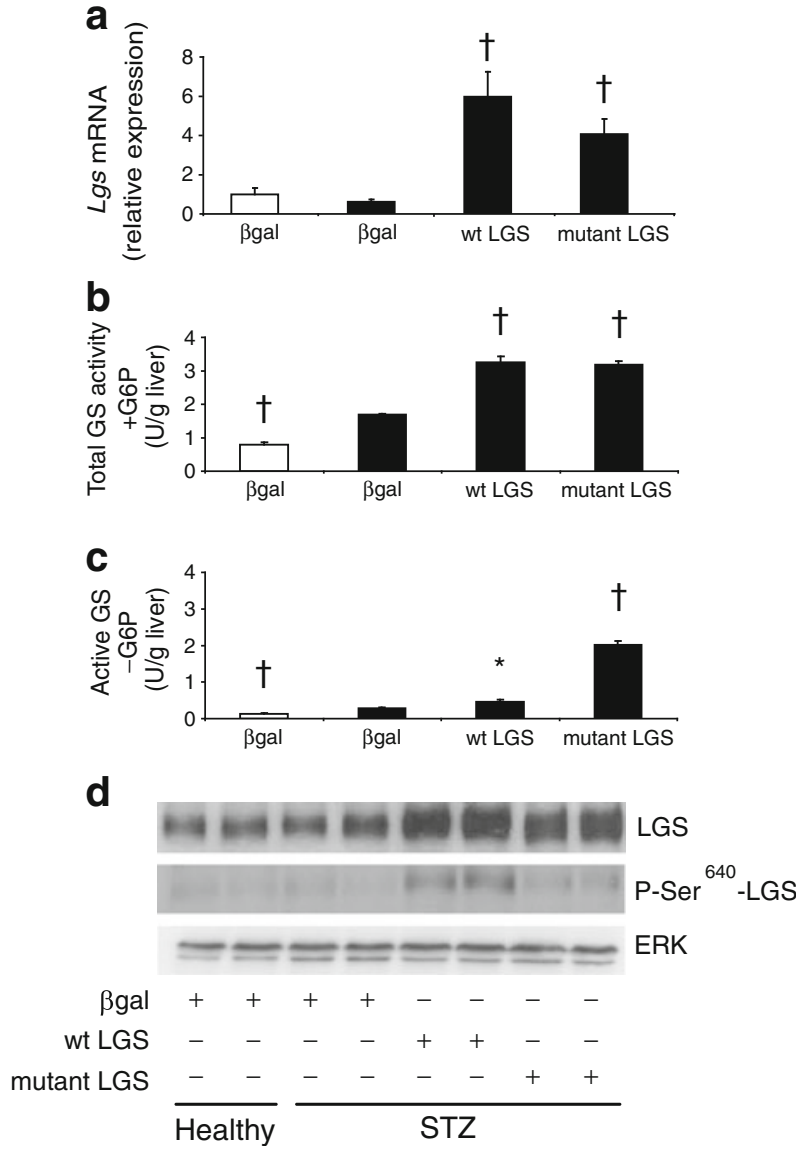

Fig. 1 Overproduction of wt-LGS or activated mutant-LGS. a RT-QPCR analysis of LGS mRNA levels in rat livers overproducing $\beta$ gal, wt-LGS or mutant-LGS. b Total GS activity of liver homogenates from the $\beta$ gal-, wt-LGS- or mutant-LGS-overproducing rat groups. c GS activity measured in the absence of G6P in these groups of rats. In all cases (a-c), data represent the mean \pm SE for six healthy $\beta$ galoverproducing rats, nine STZ $\beta$ gal-overproducing rats, six STZ wt-LGS-overproducing rats and five STZ mutant-LGS overproducing rats. Statistically significant difference for comparisons with $\beta$ gal STZ group with $* p<0.05$ or ${ }^{\dagger} p<0.005$. White bar, healthy rats; black bar, STZ-treated rats. d Representative immunoblotting analysis of liver extracts of healthy or STZ-treated rats with antibodies against total LGS, GS phosphorylated on Ser640 or the mitogen-activated protein kinase ERK as a load control

(Fig. 1c), as expected from previous in vitro and in vivo studies $[9,10]$.

Liver glycogen deposition We next examined the effect of LGS overproduction on liver glycogen stores. STZ treatment caused a significant decrease in liver glycogen content. Remarkably, the overproduction of activated mutant-LGS, but not of the wt-LGS, in liver of fed STZ rats increased liver glycogen up to levels similar to those of healthy controls (Fig. 2). After overnight starvation, the glycogen content of the group overproducing activated mutant-LGS was decreased compared with the fed state, indicating net mobilisation of liver glycogen (Fig. 2).
However, glycogen was not fully depleted, as observed in healthy, starved animals.

Effects on blood glucose and other variables Blood glucose was measured daily after adenoviral injection. STZ-injected rats that received the activated mutant-LGSencoding adenovirus showed a decrease in blood glucose that was significant $96 \mathrm{~h}$ after injection, and this decrease was greater at the final point of the experiment, at $144 \mathrm{~h}$ $(14.3 \pm 1.6$ vs $27.5 \pm 2.4 \mathrm{mmol} / \mathrm{l}$; Fig. $3 \mathrm{a})$. This reduction in blood glucose concentration was not due to an increase in circulating insulin (Table 1). Neither plasma triacylglycerol nor lactate were changed (Table 1).

Effects on food consumption Monitoring of food consumption during these studies revealed a clear effect on this variable. Animals transduced with the activated mutantLGS consumed $30 \%$ less food on a daily basis, when compared with the $\beta$ gal and wt-LGS STZ groups, which showed an almost doubled food intake compared with that of the healthy $\beta$ gal group (Fig. 3b). The reduction was already significant at $72 \mathrm{~h}$ (Fig. 3c) and it did not have an impact on body weight (Table 1). As leptin plays a major role in the control of food intake and feeding behaviour, we examined whether the reduction in food intake was secondary to changes in circulating leptin levels. While STZ injection caused a 1.7-fold decrease, overproduction of activated mutant-LGS did not affect leptin levels (Fig. 3d).

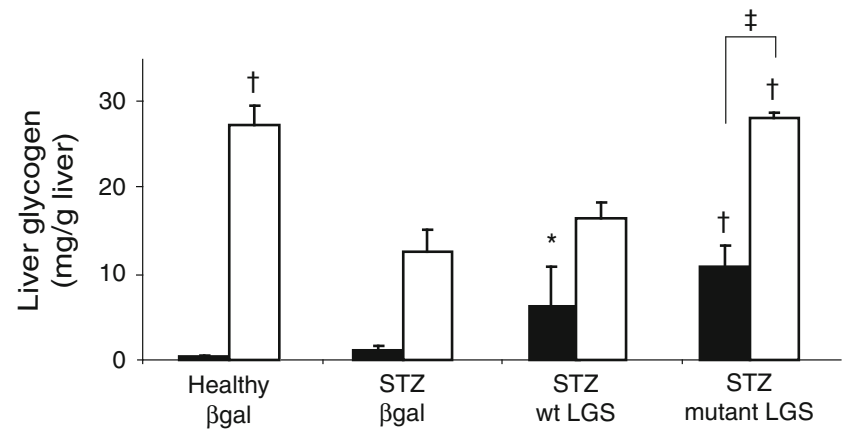

Fig. 2 Effects on glycogen content. Liver glycogen content measured in healthy, $\beta$ gal-overproducing rats or STZ-treated rats overproducing $\beta$ gal, wt-LGS or activated mutant-LGS. Liver glycogen content was also measured in a new group of rats that were starved for $18 \mathrm{~h}$. White bar, fed rats; black bar, starved rats. Data represent the mean \pm SE for six healthy $\beta$ gal-overproducing rats, nine STZ $\beta$ gal-overproducing rats, six STZ wt-LGS-overproducing rats, five STZ mutant-LGSoverproducing rats, seven starved healthy $\beta$ gal-overproducing rats, five starved STZ $\beta$ gal-overproducing rats, three starved STZ wt-LGSoverproducing rats and six starved STZ mutant-LGS-overproducing rats. Statistically significant difference for comparisons with $\beta$ gal STZ group in the same metabolic state with ${ }^{*} p<0.05$ and ${ }^{\dagger} p<0.005$. Statistically significant difference between fed and starved states for the mutant-LGS-overproducing STZ rats with ${ }^{\ddagger} p<0.005$ 
a

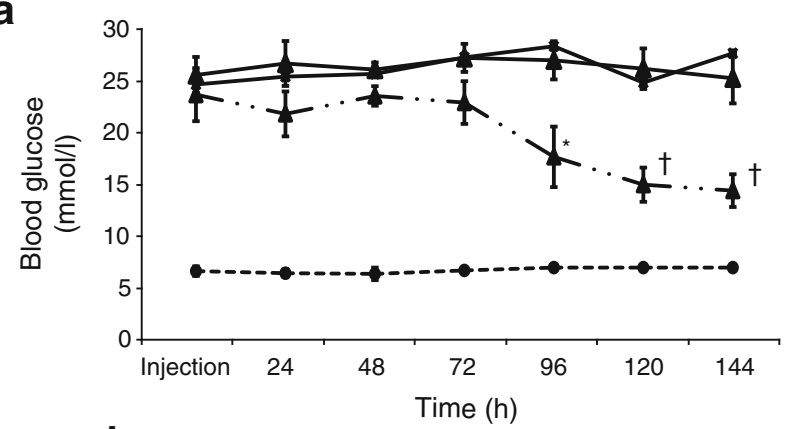

b

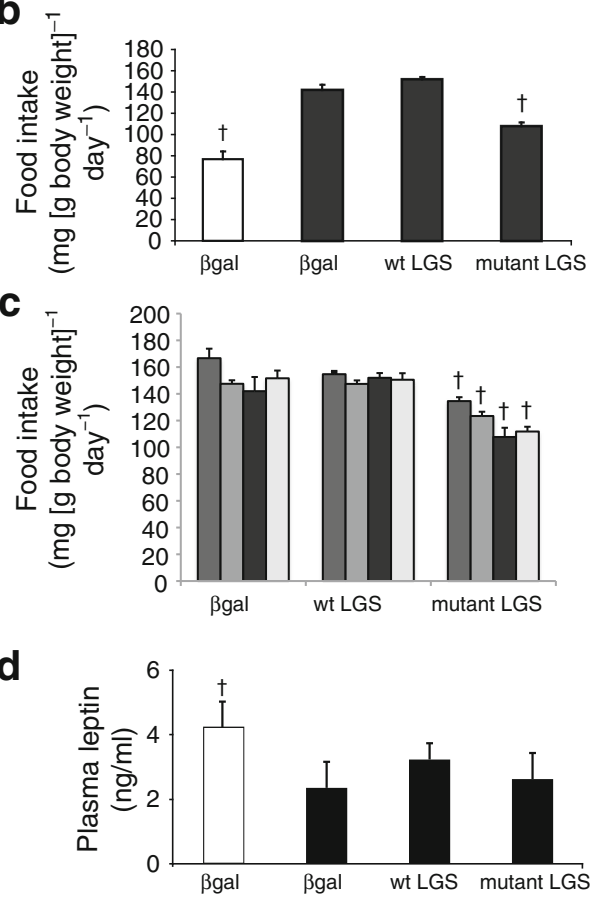

Fig. 3 Effects on blood glucose levels and food intake a Blood glucose was measured daily for $144 \mathrm{~h}$ after adenoviral injection by using tail vein blood samples. Black square, $\beta$ gal-overproducing STZ rats; black triangle with solid line, wt-LGS-overproducing STZ rats; black triangle with broken line, mutant-LGS-overproducing STZ rats; black circle, $\beta$ gal-overproducing healthy rats. b Food intake was measured during three successive $24 \mathrm{~h}$ periods, beginning 3 days after viral treatment. The graph shows food intake at $120 \mathrm{~h}$. White bar, healthy rats; black bar, STZ-treated rats. c Time course of food consumption for the STZ-treated rats. Dark grey bar, $72 \mathrm{~h}$; light grey bar, $96 \mathrm{~h}$; black bar, $120 \mathrm{~h}$; white bar, $144 \mathrm{~h}$. d Plasma leptin levels were determined by radioimmunoassay $144 \mathrm{~h}$ after viral injection. White bar, healthy rats; black bar, STZ-treated rats. In all cases, data represent the mean $\pm \mathrm{SE}$ intake $/ 24 \mathrm{~h}(\mathbf{b}, \mathbf{c})$ and ng leptin/ml plasma (d) for six $\beta$ gal-overproducing healthy rats, nine $\beta$ gal-overproducing STZ rats, six wt-LGS-overproducing STZ rats and five mutant-LGSoverproducing STZ rats. Statistically significant difference for comparisons with $\beta$ gal STZ group with ${ }^{*} p<0.05$ and ${ }^{\dagger} p<0.005$

Effects on gene expression of key proteins involved in glycogen metabolism We next studied whether the overproduction of LGS caused changes in other key enzymes and proteins involved in the control of glucose metabolism. STZ treatment produced a reduction in the mRNA levels of
$G k, G p$ (also known as $P y g l$ ) and two regulatory subunits of protein phosphatase $1(P P 1), G_{L}$ and protein targeting to glycogen (Ptg; also known as Ppp1r3c), and an increase in the mRNA levels of Glut2 (also known as Slc2a2; Fig. 4), as previously described [23-27]. In our study, the marked decrease in $G k$ mRNA and GK protein levels induced by STZ treatment was not reversed in any case, as measured by RT-Q-PCR and western blot analysis, respectively (Fig. $4 \mathrm{~b}, \mathrm{j}$ ). Neither were $G p$ mRNA or protein levels modified (Fig. 4c, j). However, we observed an increase in GP protein phosphorylated at Ser14 (corresponding to the activated GP) in STZ-treated rats overproducing activated mutant-LGS (Fig. 4j). No changes in the mRNA levels of the PP1 regulatory subunit $G_{L}$ or of Glut2 were detected in adenoviral-transduced animals (Fig. 4a, d). Interestingly, Ptg mRNA levels were further decreased in STZ-injected rats overproducing activated mutant-LGS compared with those overproducing $\beta$ gal or wt-LGS (Fig. 4e).

Effects on gene expression of key enzymes involved in gluconeogenesis Since gluconeogenesis is aberrantly activated in diabetes, we analysed whether overproduction of LGS caused changes in the production of the main gluconeogenic enzymes, namely glucose-6-phosphatase (G6Pase), fructose-1,6-bisphosphatase (FBPase), pyruvate carboxylase (PC) and PEPCK. STZ treatment caused the expected increase in the levels of G6Pase, FBPase and PEPCK, but did not affect PC in fed animals. Overproduction of activated mutant-LGS in STZ-treated rats induced a decrease in G6pase (also known as G6pc), Fbpase (also known as Fbp2) and Pepck mRNA hepatic expression, but no differences in $P c$ were found (Fig. $4 \mathrm{f}-\mathrm{i}$ ). Accordingly, PEPCK immunodetection revealed that the levels of this protein were normalised by overproduction of activated mutant-LGS (Fig. 4j).

Effects on cultured hepatocytes Finally, we examined whether the observed effects on gene expression were due to a cell-autonomous mechanism sensing the increased synthesis of glycogen or to a systemic mechanism. To this end, hepatocytes isolated from healthy rats were infected with adenoviruses coding for $\beta$ gal, wt-LGS and the activated mutant-LGS, and gluconeogenesis was induced by dexamethasone and dibutyryl cAMP treatment for $24 \mathrm{~h}$. Hepatocytes infected with these adenoviruses expressed similar amounts of the corresponding enzymes, as revealed by western blot analysis (Fig. 5a). The hepatocytes infected with the wt-LGS- or the activated mutant-LGS-encoding adenoviruses showed a similar increase in total GS activity, although only the latter showed a rise in the concentration of catalytically active GS (Fig. 5b). Induction of gluconeogenesis caused the expected increase in the expression of G6pase, Fbpase and Pepck. Interestingly, overexpression of 
Table 1 Plasma variables and body weight in healthy and STZ-treated rats overexpressing $\beta$ gal, wt-LGS and activated mutant-LGS

\begin{tabular}{|c|c|c|c|c|}
\hline Variable & Healthy $\beta$ gal $(n=6)$ & STZ $\beta$ gal $(n=9)$ & STZ wt-LGS $(n=6)$ & STZ mutant-LGS $(n=5)$ \\
\hline Glucose $(\mathrm{mmol} / \mathrm{l})$ & $7.5 \pm 0.2$ & $28 \pm 2.4^{\mathrm{a}}$ & $25 \pm 3.2^{\mathrm{a}}$ & $14 \pm 1.6^{\mathrm{b}}$ \\
\hline Insulin (pmol/1) & $241 \pm 51$ & $120 \pm 34^{\mathrm{a}}$ & $103 \pm 34^{\mathrm{a}}$ & $138 \pm 34^{\mathrm{a}}$ \\
\hline Triacylglycerol (mmol/l) & $1.6 \pm 0.3$ & $1.4 \pm 0.2$ & $1.4 \pm 0.3$ & $1.5 \pm 0.3$ \\
\hline Lactate $(\mathrm{mmol} / \mathrm{l})$ & $5 \pm 0.8$ & $6.9 \pm 0.4$ & $6.5 \pm 0.2$ & $7.5 \pm 0.7$ \\
\hline Initial weight $(\mathrm{g})$ & $235 \pm 3$ & $230 \pm 4$ & $223 \pm 4$ & $229 \pm 4$ \\
\hline Final weight $(\mathrm{g})$ & $267 \pm 13$ & $235 \pm 4^{\mathrm{a}}$ & $226 \pm 3^{a}$ & $235 \pm 5^{\mathrm{a}}$ \\
\hline
\end{tabular}

Data represent the mean $\pm \mathrm{SE}$

Blood samples were taken $144 \mathrm{~h}$ after infection with the indicated adenoviruses and used for measurement of the metabolites and hormones indicated

${ }^{a}$ Statistically significant difference for comparisons with $\beta$ gal healthy group $(p<0.01)$

${ }^{\mathrm{b}}$ Statistically significant difference for comparisons with $\beta$ gal STZ group $(p<0.05)$

the activated mutant-LGS form, but not of wt-LGS, counteracted the increase in the expression of these three genes also in cultured cells (Fig. $5 \mathrm{c}-\mathrm{e}$ ), as happened in the STZ-treated rats. In addition, Ptg mRNA levels were also reduced by the production of the activated mutant-LGS (Fig. 5f), as observed in the in vivo model.

Moreover, we tested the hypothesis that forced accumulation of an intrinsically dephosphorylated mutant form of
Fig. 4 Effects on gene expression. RT-Q-PCR analysis of Glut2 (a), Gk (b), Gp (c) and the regulatory PP1 subunits $G_{L}(\mathbf{d})$ and Ptg (e) mRNA levels in livers of fed healthy and STZtreated rats overproducing $\beta \mathrm{gal}$, and in STZ rats overproducing wt-LGS or mutant-LGS. RT-QPCR analysis of G6pase (f), Fbpase (g), Pc (h) and Pepck (i) mRNA levels in livers of all groups. Data show mRNA levels relative to those in healthy rats overproducing $\beta$ gal and represent the mean $\pm \mathrm{SE}$ for six $\beta$ gal-overproducing healthy rats, nine $\beta$ gal-overproducing STZ rats, six wt-LGSoverproducing STZ rats, and five mutant (mut.)-LGS-overproducing STZ rats. $\mathbf{j}$ Immunoblotting analysis of liver extracts of healthy or STZ rats with antibodies against GK, phosphorylated-GP (active), GP, PEPCK or with an antibody against the mitogen-activated protein kinase ERK as a loading control. The phosphorylated-GP vs total GP ratio is shown in the side graph. White bar, healthy rats; black bar, STZ-treated rats. Statistically significant difference for comparisons with $\beta$ gal STZ group with $* p<0.05$ and ${ }^{\dagger} p<0.005$
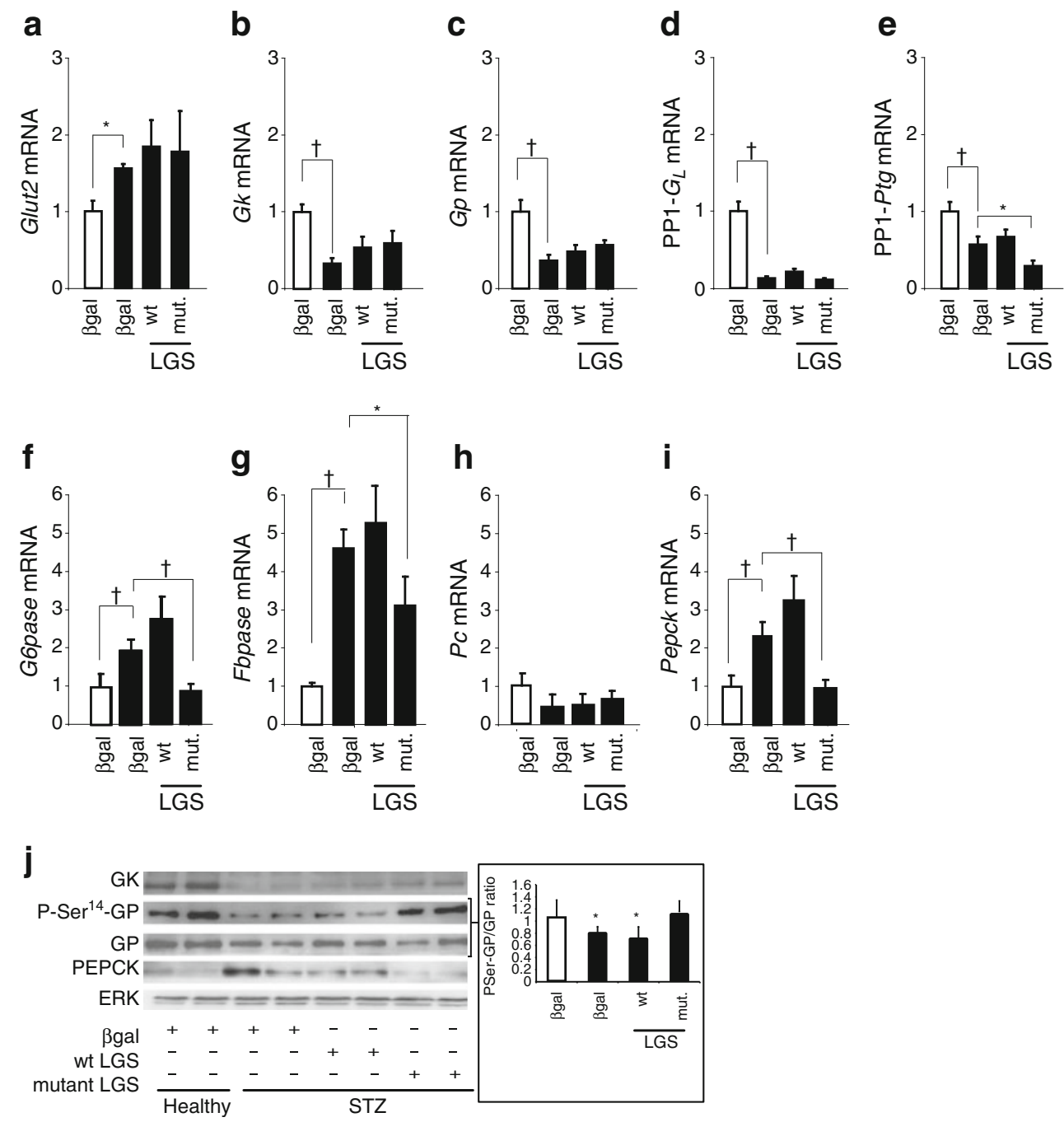


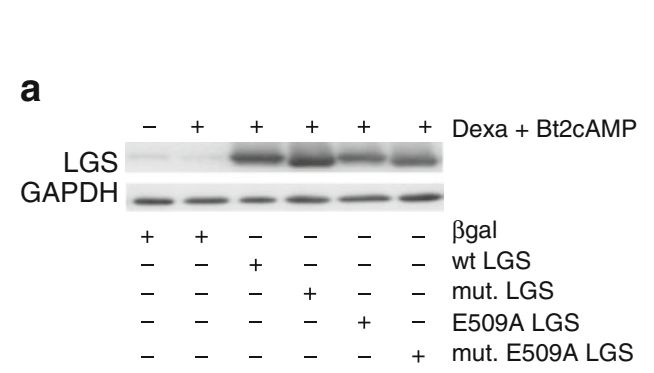

C

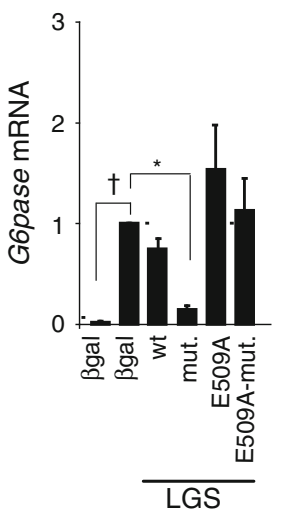

d

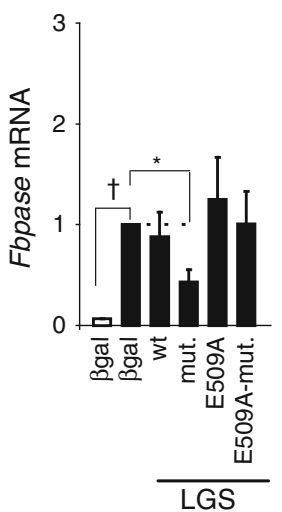

Fig. 5 Effects of activated mutant (mut.)-LGS overproduction in cultured hepatocytes. a Representative western blot analysis of homogenates from cultured rat hepatocytes overproducing $\beta$ gal, wtLGS, activated mutant-LGS, E509A-LGS (catalytically dead mutant) or E509A-mutant-LGS (catalytically dead mutant with Ser 2 and $3 \mathrm{~b}$ to Ala mutations), and treated with $100 \mathrm{nmol} / 1$ dexamethasone and $0.3 \mathrm{mmol} / 1$ dibutyryl cAMP (Bt2cAMP) for $24 \mathrm{~h}$, and from untreated hepatocytes overproducing $\beta$ gal. Bands were detected using an antibody against LGS or an antibody against glyceraldehyde-3phosphate dehydrogenase (GAPDH) as a control of charge. b Total GS activity, measured in the presence of G6P (black bars), and active GS activity, measured in the absence of G6P (white bars), of

the LGS protein rather than increased LGS activity was the cause of the effects observed on gene expression. To this end, we included in the in vitro experiment the infection with adenoviruses coding for a catalytically inactive mutant (E509A-LGS) [13] and an inactive, non-phosphorylatable LGS form (E509A-LGS with Ser to Ala mutations at sites 2 and 3B, named E509A-mutant-LGS) [9]. While the level of these mutant forms was similar to that observed for the wtLGS and the activated mutant-LGS (Fig. 5a), there was no increase in total GS activity compared with $\beta$ galtransduced hepatocytes (Fig. 5b). No changes were found in the expression of the genes tested (G6pase, Fbpase, Pepck and Ptg) in hepatocytes overproducing E509A-LGS or E509A-mutant-LGS proteins on induction of gluconeogenesis (Fig. 5c-f). These observations confirm that glycogen synthesis is required for the blockade of gluconeogenic genes.
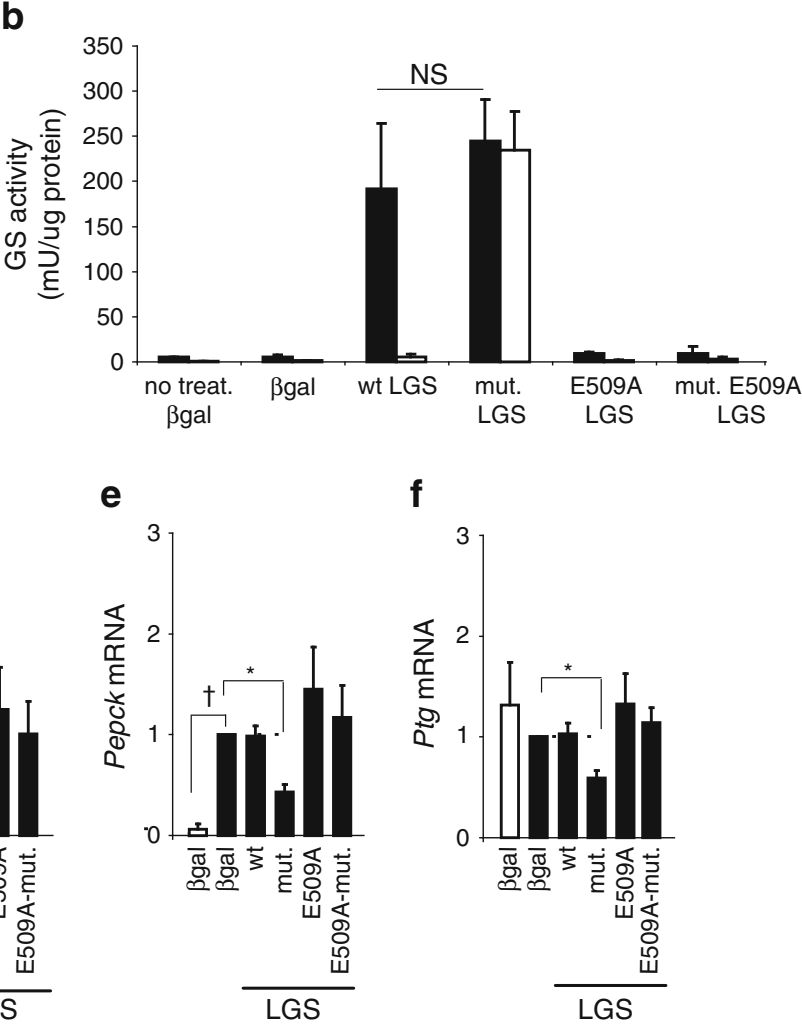

homogenates from hepatocytes overexpressing the above-described proteins. Data represent the mean $\pm \mathrm{SE}$ for four to six independent experiments. NS, no statistically significant difference in the total GS activity of wt-LGS- and mutant-LGS-overexpressing hepatocytes. RTQ-PCR analysis of Grpase (c), Fbpase (d), Pepck (e) and Ptg (f) mRNA levels in these hepatocytes. Data show mRNA levels relative to those in healthy rats overproducing $\beta$ gal and represent the mean \pm SE for six independent experiments. Statistically significant difference for comparisons with ßgal-overproducing-, dexamethasone (Dex)/ Bt2cAMP-treated hepatocytes with ${ }^{*} p<0.05$ or ${ }^{\dagger} p<0.005$. White bars, no treatment; black bars, Dex/Bt2cAMP-treated

\section{Discussion}

The liver plays a major role in the clearance of blood glucose in the postprandial state [28], and several proteins involved in the control of hepatic glycogen metabolism, including GK, GP, GSK-3 and glycogen targeting proteins, have been proposed as potential targets for anti-hyperglycaemic therapy for diabetes. In fact, most of these strategies lead to secondary activation of LGS (by reducing its phosphorylation) and to increased glycogen deposition. However, each of these approaches results in side effects on other metabolic pathways, complicating the interpretation of the results and limiting their therapeutic use. We have recently demonstrated that the overabundance of a constitutively active form of LGS in liver of healthy rats improves glucose tolerance in the fed state without compromising glycogenolysis in the postabsorptive state [10]. Therefore, the aim of this study 
was to determine the direct effects of activation of LGS as the primary target for diabetes therapy, a strategy that has not previously been addressed. Several conclusions can be drawn from this study.

First, only activated mutant-LGS overproduction in the liver of STZ rats, and not wt-LGS overproduction, was sufficient to restore the impaired capacity of the diabetic liver to accumulate glycogen. Moreover, glycogen accumulated in livers producing activated mutant-LGS was mobilised in response to starvation. This observation indicates that the glycogen-disposal mechanism is functional in this scenario. Diverting the excess of glucose in blood towards the synthesis of this polysaccharide in the liver can only partially account for the decrease in blood glucose observed in STZ-treated rats overproducing an activated mutant-LGS form, since on a quantitative basis the amount of glucose that can be accumulated as glycogen is limited. Importantly, the decline in blood glucose levels was produced without any alteration in triacylglycerols and lactate within the context of diminished circulating insulin associated with the diabetic state.

Second, another possible mechanism contributing to the lowering of blood glucose in animals with hepatic activated mutant-LGS production is the decrease in food intake. STZinduced diabetes caused a decrease in circulating leptin levels, and it has been reported that leptin infusion results in normalisation of food intake and a moderate reduction in glycaemia [29]. However, our results show that leptin levels were not affected by activated mutant-LGS overproduction in STZ-treated rats. Thus, although we cannot rule out that levels of other signalling peptides could be altered by activated mutant-LGS overproduction, it is also conceivable that the reduction in food intake is related to changes in glucose availability and its storage, supporting the idea of a 'glycogenostatic' model in which hepatic glycogen stores play a central role in regulating food intake and energy balance, as proposed by other groups [30-35]. Remarkably, in terms of overall carbohydrate balance, the reduction in food intake was more relevant than the increase in glucose diverted to hepatic glycogen. Our work supports the notion that both the glycogenic potential and a full sensitivity to glycogenolytic signals are necessary to keep food intake under check, as previously suggested by other studies [8].

Third, activated mutant-LGS overproduction in liver of STZ-injected rats produced the restoration of GP phosphorylation, a variable known to be altered in diabetes [26]. Winnick and collaborators have very recently reported that hepatic glycogen supercompensation induces an increase in GP activity [36]. A similar compensatory effect to limit glycogen deposition may account for the observed increase in GP phosphorylation in activated mutant-LGS-treated diabetic rats. This effect may be mediated, among other signals, by the observed reduction in PTG transcription caused by active-LGS overproduction.
Last, but not least, our results show that mRNA levels of the main enzymes involved in the control of gluconeogenesis, namely FBPase, G6Pase and PEPCK [37], were decreased in the liver of STZ-treated rats overproducing the activated mutant-LGS form compared with control diabetic animals. In this regard, it is worth noting that selective GSK-3 inhibitors, which promote the activation of GS, have also been reported to increase hepatic glycogen synthesis and decrease hepatic glucose output in Zucker diabetic fatty ( falfa) rats $[38,39]$. These compounds also cause a reduction in the levels of gluconeogenic enzymes, PEPCK and G6Pase, in hepatoma cell lines [40, 41]. Moreover, it has been recently described that $L g s$ (also known as Gys2) knockout mice have decreased and delayed suppression of gluconeogenesis [42]. Taken together, these data reinforce the idea that LGS activity plays a central role in the control of the gluconeogenic programme. Importantly, our results indicate that the changes in gluconeogenic gene expression were due directly to the increase in LGS activity and thus to glycogen deposition, and not to the accumulation of the dephosphorylated mutant protein, since the production of a catalytically dead variant of this mutant did not reproduce its effects on gene expression. In addition, here we show that the control of gluconeogenic gene expression is an insulin-independent, cell-autonomous mechanism, since it occurred in the context of an insulindeficient state in vivo and was reproduced in isolated hepatocytes. Notably, the changes in gluconeogenic gene expression described here may contribute to a decrease in hepatic glucose production, which is one of the main targets of diabetes therapy. Furthermore, the 'pulling' effect of activated-mutant LGS may also contribute to increasing the number of glucose molecules from the hepatic G6P pool directed into glycogen, thus preventing it from being released as glucose into the blood.

In conclusion, the restoration of impaired liver glycogen deposition by overproduction of an active form of LGS in diabetic STZ-treated rats reversed hyperglycaemia by the following three mechanisms: (1) incorporating blood glucose into hepatic glycogen; (2) decreasing hyperphagia; and (3) reducing the production of the main gluconeogenic enzymes. Our results support the idea that LGS activation may provide an effective therapy for diabetes.

Acknowledgements S. Ros was the recipient of a predoctoral fellowship from the Ministerio de Educación y Ciencia (Spain). J. Calbó is the recipient of an Intra-European Fellowship from the Marie Curie Actions (FP7-PEOPLE-2009-IEF). This work was supported by grants BFU2008-00769 and SAF2007-64722 (Ministry of Science and Innovation, Spain), 2009SGR-1176 (Autonomous Government of Catalonia) and a grant from the Fundación Marcelino Botín to J.J. Guinovart. CIBER de Diabetes y Enfermedades Metabólicas Asociadas is an ISCIII project. We thank the Animal Research Centre of the Barcelona Science Park for their help with the assessment and equipment necessary to carry out this study. We thank D. Zafra (IRB Barcelona) for her help in the 
preparation of the revised version of the manuscript. We are also grateful to A. Adrover, L. Babin and E. Veza for their technical support. We thank T. Yates (permanent full-time employee of IRB Barcelona) for correcting the English version of the manuscript and L. Agius, University of Newcastle, for helpful suggestions.

Contribution statement S.R. contributed to the conception, design and analysis of the data, and to drafting the manuscript. M.G.R. contributed to the conception and analysis of the data and critically revised the manuscript. J.C. analysed and interpreted the data and contributed to drafting the article. J.J.G. directed the study, and contributed to the conception of the experiments, interpretation of the data and writing the paper. All authors provided final approval of the version of the manuscript to be published.

Duality of interest The authors declare that there is no duality of interest associated with this manuscript.

\section{References}

1. Roach PJ, Cheng C, Huang D et al (1998) Novel aspects of the regulation of glycogen storage. J Basic Clin Physiol Pharmacol 9:139-151

2. Magnusson I, Rothman DL, Katz LD, Shulman RG, Shulman GI (1992) Increased rate of gluconeogenesis in type II diabetes mellitus. $\mathrm{A}{ }^{13} \mathrm{C}$ nuclear magnetic resonance study. J Clin Invest 90:1323-1327

3. Cline GW, Rothman DL, Magnusson I, Katz LD, Shulman GI (1994) ${ }^{13}$ C-nuclear magnetic resonance spectroscopy studies of hepatic glucose metabolism in normal subjects and subjects with insulin-dependent diabetes mellitus. J Clin Invest 94:23692376

4. Velho G, Petersen KF, Perseghin G et al (1996) Impaired hepatic glycogen synthesis in glucokinase-deficient (MODY-2) subjects. J Clin Invest 98:1755-1761

5. O'Doherty RM, Lehman DL, Telemaque-Potts S, Newgard CB (1999) Metabolic impact of glucokinase overexpression in liver: lowering of blood glucose in fed rats is accompanied by hyperlipidemia. Diabetes 48:2022-2027

6. Baker DJ, Greenhaff PL, MacInnes A, Timmons JA (2006) The experimental type 2 diabetes therapy glycogen phosphorylase inhibition can impair aerobic muscle function during prolonged contraction. Diabetes 55:1855-1861

7. Gasa R, Clark C, Yang R, DePaoli-Roach AA, Newgard CB (2002) Reversal of diet-induced glucose intolerance by hepatic expression of a variant glycogen-targeting subunit of protein phosphatase-1. J Biol Chem 277:1524-1530

8. Yang R, Newgard CB (2003) Hepatic expression of a targeting subunit of protein phosphatase-1 in streptozotocin-diabetic rats reverses hyperglycemia and hyperphagia despite depressed glucokinase expression. J Biol Chem 278:23418-23425

9. Ros S, Garcia-Rocha M, Dominguez J, Ferrer JC, Guinovart JJ (2009) Control of liver glycogen synthase activity and intracellular distribution by phosphorylation. J Biol Chem 284:6370-6378

10. Ros S, Zafra D, Valles-Ortega J et al (2010) Hepatic overexpression of a constitutively active form of liver glycogen synthase improves glucose homeostasis. J Biol Chem 285:37170-37177

11. Gomis RR, Ferrer JC, Guinovart JJ (2000) Shared control of hepatic glycogen synthesis by glycogen synthase and glucokinase. Biochem J 351:811-816

12. Becker TC, Noel RJ, Coats WS et al (1994) Use of recombinant adenovirus for metabolic engineering of mammalian cells. Methods Cell Biol 43:161-189
13. Cid E, Gomis RR, Geremia RA, Guinovart JJ, Ferrer JC (2000) Identification of two essential glutamic acid residues in glycogen synthase. J Biol Chem 275:33614-33621

14. Massague J, Guinovart JJ (1977) Insulin control of rat hepatocyte glycogen synthase and phosphorylase in the absence of glucose. FEBS Lett 82:317-320

15. Thomas JA, Schlender KK, Larner J (1968) A rapid filter paper assay for UDPglucose-glycogen glucosyltransferase, including an improved biosynthesis of UDP- ${ }^{14} \mathrm{C}$-glucose. Anal Biochem 25:486-499

16. Chan TM, Exton JH (1976) A rapid method for the determination of glycogen content and radioactivity in small quantities of tissue or isolated hepatocytes. Anal Biochem 71:96-105

17. Garcia-Rocha M, Roca A, de la Iglesia N et al (2001) Intracellular distribution of glycogen synthase and glycogen in primary cultured rat hepatocytes. Biochem J 357:17-24

18. de la Iglesia N, Veiga-da-Cunha M, van Schaftingen E, Guinovart JJ, Ferrer JC (1999) Glucokinase regulatory protein is essential for the proper subcellular localisation of liver glucokinase. FEBS Lett 456:332-338

19. Gomis RR, Cid E, Garcia-Rocha M, Ferrer JC, Guinovart JJ (2002) Liver glycogen synthase but not the muscle isoform differentiates between glucose 6-phosphate produced by glucokinase or hexokinase. J Biol Chem 277:23246-23252

20. Gomis RR, Favre C, Garcia-Rocha M, Fernandez-Novell JM, Ferrer JC, Guinovart JJ (2003) Glucose 6-phosphate produced by gluconeogenesis and by glucokinase is equally effective in activating hepatic glycogen synthase. J Biol Chem 278:9740-9746

21. Trinh KY, O'Doherty RM, Anderson P, Lange AJ, Newgard CB (1998) Perturbation of fuel homeostasis caused by overexpression of the glucose-6-phosphatase catalytic subunit in liver of normal rats. J Biol Chem 273:31615-31620

22. Herz J, Gerard RD (1993) Adenovirus-mediated transfer of low density lipoprotein receptor gene acutely accelerates cholesterol clearance in normal mice. Proc Natl Acad Sci USA 90:2812-2816

23. Burcelin R, Eddouks M, Kande J, Assan R, Girard J (1992) Evidence that GLUT-2 mRNA and protein concentrations are decreased by hyperinsulinaemia and increased by hyperglycaemia in liver of diabetic rats. Biochem J 288:675-679

24. Doherty MJ, Cadefau J, Stalmans W, Bollen M, Cohen PT (1998) Loss of the hepatic glycogen-binding subunit (GL) of protein phosphatase 1 underlies deficient glycogen synthesis in insulindependent diabetic rats and in adrenalectomized starved rats. Biochem J 333:253-257

25. Browne GJ, Delibegovic M, Keppens S, Stalmans W, Cohen PT (2001) The level of the glycogen targetting regulatory subunit R5 of protein phosphatase 1 is decreased in the livers of insulindependent diabetic rats and starved rats. Biochem J 360:449-459

26. Rao PV, Pugazhenthi S, Khandelwal RL (1995) The effects of streptozotocin-induced diabetes and insulin supplementation on expression of the glycogen phosphorylase gene in rat liver. J Biol Chem 270:24955-24960

27. Iynedjian PB (1993) Mammalian glucokinase and its gene. Biochem J 293:1-13

28. Cherrington AD (1999) Banting Lecture, 1997. Control of glucose uptake and release by the liver in vivo. Diabetes 48:1198-1214

29. Sindelar DK, Havel PJ, Seeley RJ, Wilkinson CW, Woods SC, Schwartz MW (1999) Low plasma leptin levels contribute to diabetic hyperphagia in rats. Diabetes 48:1275-1280

30. Mayer J (1953) Glucostatic mechanism of regulation of food intake. N Engl J Med 249:13-16

31. Russek M (1963) Participation of hepatic glucoreceptors in the control of intake of food. Nature 197:79-80

32. Tordoff MG, Friedman MI (1988) Hepatic control of feeding: effect of glucose, fructose, and mannitol infusion. Am J Physiol 254:R969-R976 
33. Tordoff MG, Tluczek JP, Friedman MI (1989) Effect of hepatic portal glucose concentration on food intake and metabolism. Am J Physiol 257:R1474-R1480

34. Mayer J (1991) Bulletin of the New England Medical Center, Volume XIV, April-June 1952: the glucostatic theory of regulation of food intake and the problem of obesity (a review). Nutr Rev 49:46-48

35. Flatt JP (1996) Carbohydrate balance and body-weight regulation. Proc Nutr Soc 55:449-465

36. Winnick JJ, An Z, Ramnanan CJ et al (2011) Hepatic glycogen supercompensation activates AMP-activated protein kinase, impairs insulin signaling, and reduces glycogen deposition in the liver. Diabetes 60:398-407

37. Granner D, Pilkis S (1990) The genes of hepatic glucose metabolism. J Biol Chem 265:10173-10176

38. Cline GW, Johnson K, Regittnig W et al (2002) Effects of a novel glycogen synthase kinase-3 inhibitor on insulin-stimulated glu- cose metabolism in Zucker diabetic fatty $(f a / f a)$ rats. Diabetes 51:2903-2910

39. Ring DB, Johnson KW, Henriksen EJ et al (2003) Selective glycogen synthase kinase 3 inhibitors potentiate insulin activation of glucose transport and utilization in vitro and in vivo. Diabetes 52:588-595

40. Lochhead PA, Coghlan M, Rice SQ, Sutherland C (2001) Inhibition of GSK-3 selectively reduces glucose-6-phosphatase and phosphatase and phosphoenolypyruvate carboxykinase gene expression. Diabetes 50:937-946

41. Coghlan MP, Culbert AA, Cross DA et al (2000) Selective small molecule inhibitors of glycogen synthase kinase-3 modulate glycogen metabolism and gene transcription. Chem Biol 7:793-803

42. Irimia JM, Meyer CM, Peper CL et al (2010) Impaired glucose tolerance and predisposition to the fasted state in liver glycogen synthase knock-out mice. J Biol Chem 285:12851-12861 